\title{
.
}

José Luis Rodríguez López*

Pedro Hinojo González ${ }^{* *}$

\section{OPORTUNIDADES DE LA TECNOLOGÍA APLICADA A LOS MERCADOS FINANCIEROS: EL FENÓMENO FINTECH}

El fenómeno Fintech se puede definir como la aplicación disruptiva de las nuevas tecnologías de la información y las comunicaciones al sistema financiero. Estas innovaciones, impulsadas por una conjunción de factores tecnológicos y socioeconómicos, están alterando el marco competitivo en el sector y generan nuevas oportunidades y modelos de negocio. Los principales efectos son un aumento de la competencia y una potencial mitigación de ciertos fallos de mercado, como las imperfecciones de la información, que han afectado tradicionalmente al sistema financiero.

Palabras claves: Fintech, innovación, tecnología, mercados financieros, servicios financieros, competencia.

Clasificación JEL: D53, G10, G20, K23.

\section{Introducción}

El fenómeno Fintech suscita enorme interés y da lugar a vivos debates acerca de sus determinantes y sus posibles efectos. A día de hoy y dada su relativa novedad, el fenómeno Fintech carece de una definición universalmente

\footnotetext{
* Jefe de Área de la Subdirección de Estudios e Informes. Departamento de Promoción de la Competencia de la CNMC.

** Vocal Asesor de la Subdirección de Estudios e Informes. Departamento de Promoción de la Competencia de la CNMC.

Versión de febrero de 2019.

Las opiniones expresadas en el presente artículo son de los autores. Los autores desean agradecer las aportaciones de Lara Tobías Peña, María Sobrino Ruiz y Gilmer Sánchez Valenzuela. Los errores $u$ omisiones que pudieran existir son en cualquier caso responsabilidad exclusiva de los autores.

DOI: https://doi.org/10.32796/bice.2019.3109.6777
}

aceptada, lo que puede generar cierta confusión en dichos debates. Por lo tanto, se hace obligado como primer paso dar una definición del mismo siempre que se quiera entablar un diálogo alrededor de este fenómeno. Ahí va la nuestra: Fintech consiste en la aplicación disruptiva de las nuevas tecnologías de la información y las telecomunicaciones (TIC) al sistema financiero (CNMC, 2018).

Sobre la base de esta definición se estructurará este artículo, a fin de ofrecer una perspectiva amplia y con cierta profundidad del fenómeno. El segundo apartado pretende ampliar la caracterización del fenómeno Fintech adentrándose en su delimitación (positiva y negativa, así como la clasificación de agentes que $D$ 
lo integran), su carácter disruptivo en el contexto de la industria financiera, sus factores de desarrollo y en las oportunidades que brinda. El tercer apartado aborda las distintas innovaciones sectoriales que se han considerado más relevantes: neobancos, tecnologías de registro distribuido (como blockchain), sistemas de pagos, asesoramiento financiero, financiación participativa (crowdfunding) y seguros (Insurtech). El cuarto y último apartado recoge las principales conclusiones, incluyendo las implicaciones de política económica.

\section{Factores y oportunidades subyacentes al fenómeno Fintech}

\subsection{Definición y delimitación del fenómeno Fintech}

Recordemos la definición de Fintech elegida: la aplicación disruptiva de las nuevas tecnologías de la información y las telecomunicaciones (TIC) al sistema financiero. Dado su limitado grado de concreción, a esta delimitación positiva se podría unir otra de tipo negativo: qué no consideramos Fintech. Pues bien, aquellas innovaciones «incrementales» que introducen las TIC en la forma tradicional de hacer los negocios, como la banca móvil y online (BIS, 2018), pero que no suponen una disrupción en los modelos de negocio, no se considerarán «fintech» a efectos de este análisis. Igualmente se impone realizar una aclaración sobre el uso del término, pues Fintech abarca en realidad sendas alternativas de desarrollo para los actores privados. En concreto, aparte de Fintech en un sentido amplio se habla de FinTech en un sentido restringido y su imagen especular, TechFin, sin olvidar su aplicación a la regulación y supervisión financiera o RegTech.
En efecto, Fintech en sentido amplio comprende dos estrategias alternativas seguidas por las empresas que aplican disruptivamente las nuevas TIC al negocio financiero para atender necesidades no satisfechas o mejorar la forma en que se satisfacen actualmente algunas necesidades. Por un lado, aparece lo que suele denominarse FinTech en sentido estricto, referido tanto a empresas financieras ya instaladas como a los entrantes que sean empresas de reciente creación nacidas para operar directamente en el sector financiero. Por otro lado, se tiene lo que se conoce como TechFin para referirse a aquellas empresas ya existentes que provienen de fuera del sector financiero, habitualmente del propio sector tecnológico que desarrolla las TIC, como las denominadas BigTech (Google, Amazon, Facebook, Apple...), y que extienden su negocio al sector financiero. Es decir, aprovechan su acceso y experiencia en la explotación de datos de sus clientes, a menudo en otros ámbitos, para aplicarlo al negocio financiero. En este análisis se usará el término Fintech en su definición amplia.

Asimismo, los nuevos desarrollos tecnológicos también pueden aplicarse, en el ámbito financiero, a cuestiones regulatorias. Es lo que se conoce como RegTech o «el uso de tecnología, particularmente tecnología de la información [...] en el contexto de la supervisión regulatoria, informes y cumplimiento» (Arner, Barberis y Buckley, 2017). En este sentido, la aplicación de las TIC a la regulación puede ser llevada a cabo tanto por las empresas financieras reguladas, que persiguen menores costes de cumplimiento de una mayor regulación, como por reguladores y supervisores que persiguen una aplicación y supervisión más eficiente de esta regulación financiera compleja. 


\subsection{Fintech como disrupción en el proceso de innovación financiera}

Ahora bien, un elemento clave de la definición de Fintech seguida es «disrupción», por lo que se plantea la cuestión: ¿cuán disruptivo es el fenómeno Fintech? Para responder a esta pregunta y entender la relevancia del fenómeno se necesita un enfoque general más amplio de la actividad financiera.

La industria financiera (considerada en su sentido más amplio), como toda actividad productiva, trata de satisfacer una serie de necesidades, en este caso de oferentes y demandantes últimos de fondos. En la combinación de recursos o insumos que realice, que podríamos denominar «tecnología financiera», para atender dichas necesidades, la información será el recurso clave.

En efecto, cualquier instrumento financiero (desde un depósito bancario hasta un contrato de seguro) representa en esencia una promesa de pago o reembolso futuro, entre una parte acreedora (o que ostenta el derecho de cobro o activo) y una deudora (o que contrae la obligación de pago o pasivo). Por esta naturaleza fiduciaria o de esperanza de cumplimiento de un compromiso futuro, todo instrumento financiero incorpora un significativo grado de incertidumbre, en mayor o menor grado1. Esto obliga a los agentes a incorporar y analizar constantemente toda la información disponible a fin de valorar adecuadamente cualquier instrumento financiero, toda vez que su valor será, en definitiva, pura expectativa.

En este contexto de incertidumbre sobre el cumplimiento de los compromisos de pago

1 Esta incertidumbre es mayor en unos casos (por ejemplo, en un contrato de seguro, en que el pago se suele supeditar a la ocurrencia de cierto suceso aleatorio o contingencia) que en otros (por ejemplo, un bono cuyo reembolso se realiza en un momento y condiciones predeterminadas). futuro, la situación se complica con la presencia de asimetrías informativas, tanto ex ante como ex post ${ }^{2}$, que entorpecen el buen funcionamiento de los mercados financieros. Estas asimetrías de la información exacerban la necesidad de nueva y veraz información cuya carencia puede impedir que muchos intercambios mutuamente beneficiosos lleguen a realizarse, esto es, puede llevar a situaciones subóptimas.

En este contexto, el sector financiero ofrece un claro ejemplo de solución privada a un fallo de mercado. En efecto, esta situación subóptima deja margen para que los agentes privados reduzcan eficientemente los costes de transacción (Benston y Smith, 1976) en estos mercados financieros. Esto es, se puede buscar la mitigación rentable (mediante una actividad empresarial remunerada) de las asimetrías existentes en estos mercados, en especial las asimetrías de información (Freixas y Rochet, 2008) $)^{3}$. En concreto, determinados agentes, llamados intermediarios financieros, se especializan en la actividad productiva de recopilar y analizar información sobre contrapartes, así como en vigilarlas y seleccionarlas con el objeto de minimizar los problemas de selección adversa y riesgo moral, de modo que oferentes $y$ demandantes últimos de fondos pudieran delegar en ellos estas actividades (Diamond, 1984).

La innovación financiera y el desarrollo de nuevos agentes, herramientas e instrumentos han permitido desde hace siglos, no $\sin \triangleright$

\footnotetext{
2 Por una parte, el acreedor no conoce de antemano con certeza la "calidad" o probabilidad de cumplimiento de la obligación por parte del deudor (asimetría informativa ex ante susceptible de generar situaciones de selección adversa). Por otra parte, el acreedor tendrá dificultad para determinar cuán diligente ha sido el deudor y, por ende, en qué grado el (in)cumplimiento de la promesa de pago es fruto de su esfuerzo y buena fe o del puro azar (asimetría informativa ex post susceptible de generar situaciones de riesgo moral).

3 Junto a las asimetrías informativas aparecen las denominadas asimetrías de emparejamiento (matching), originadas por indivisibilidades en los productos financieros que causan un desajuste (en tres vertientes: tamaño, plazo y riesgo) entre la variedad de producto que necesitan los demandantes y la que ofrecen los oferentes.
} 
significativos fallos y costes en muchas ocasiones, mejorar la explotación de la información disponible para una mejor valoración de productos financieros. En este sentido, Fintech puede contemplarse como un episodio más en el empeño por minimizar, de manera productiva, asimetrías informativas mediante una explotación eficiente de toda la información disponible con objeto de valorar adecuadamente los productos financieros.

No debe entenderse que Fintech es una herramienta al servicio únicamente de intermediarios financieros, sean ya conocidos (incumbentes) o nuevos (entrantes), sean FinTech (en sentido estrecho) o TechFin, sino que potencialmente puede servir incluso para reducir la necesidad de recurrir a estos intermediarios. Esto es, Fintech podría en principio permitir una mayor desintermediación de los mercados financieros de manera que oferentes y demandantes últimos puedan intercambiar productos financieros de manera directa. Un ejemplo de esto sería las plataformas de crowdfunding y crowdlending o el uso de ciertas herramientas de ayuda a la gestión de carteras y patrimonios.

\subsection{Factores determinantes en la aparición del fenómeno Fintech}

La emergencia del fenómeno Fintech (en sentido amplio) se ha visto favorecida y desencadenada por una serie de factores. En primer lugar, las recientes innovaciones tecnológicas en ciertas áreas de las TIC, especialmente inteligencia artificial y big data, computación distribuida como el registro distribuido o blockchain, criptografía y acceso móvil a internet, todas ellas con carácter mutuamente reforzado (FMI, 2017). Como se ha señalado, si la información es el insumo clave de la industria financiera, cualquier innovación que permita una mejor explotación de la misma siempre será bienvenida.

En segundo lugar, el fenómeno Fintech se ha producido de manera casi simultánea a un fenómeno más amplio, la conocida como economía colaborativa (sharing economy) y, de su mano, la aparición de los servicios bajo demanda. En efecto, la digitalización de la prestación de ciertos servicios ha revelado que existen grandes oportunidades para una mejor prestación de los mismos a través de su individualización o personalización, toda vez que una mayor diferenciación de productos permite una mejor satisfacción de las necesidades. Esta personalización de servicios puede aplicarse de manera natural al sector financiero, pues el cumplimiento de una obligación financiera depende de factores altamente idiosincráticos o subjetivos de los agentes involucrados.

En tercer lugar, y de manera, en apariencia, paradójica, la pasada crisis financiera mundial también habría fomentado el desarrollo de Fintech debido a un doble «descrédito» de muchas entidades financieras tradicionales. Por una parte, el «descrédito» en el sentido del cierre de las fuentes de financiación tradicionales a gran parte de empresas y hogares, especialmente la bancaria, derivado del credit crunch. Esta situación habría promovido la búsqueda por parte de empresas y hogares de agentes e instrumentos de financiación menos convencionales o no tradicionales (como el shadow banking o banca alternativa en la que estarían incluidas ciertas actividades de Fintech no reguladas), cuya estabilidad y eficiencia están aún por demostrar, pero que constituyen una fuente de financiación alternativa creciente en muchos países desarrollados. Y, por otra parte, la crisis financiera generó el descrédito en el sentido de desconfianza hacia muchos establecimientos financieros tradicionales e incluso del sistema financiero $D$ 
tradicional en su conjunto. Como se ha apuntado, la confianza es esencial en toda relación financiera, no solo de los intermediarios respecto de sus clientes, sino también de los proveedores de fondos respecto de los intermediarios que los demandan, de modo que el experimento que supone Fintech en el contexto postcrisis no habría encontrado entre el público el nivel de suspicacia o recelo que hubiese despertado inmediatamente antes de la crisis, cuando los intermediarios financieros tradicionales funcionaban más o menos satisfactoriamente.

$Y$ en cuarto y último lugar, la enorme regulación financiera que soporta el sector (uno de los sectores más regulados de cualquier economía desarrollada), aumentada tras la reciente crisis, puede haber incentivado la innovación disruptiva, tanto de manera directa como indirecta. De manera directa, pues esta fuerte regulación financiera constituye una poderosa barrera de entrada para los potenciales entrantes en respuesta a la señal del beneficio, lo que estimula toda innovación que permita participar en esos beneficios esquivando las barreras regulatorias. $Y$ de manera indirecta, pues la minorada tensión competitiva causada por las barreras regulatorias podría haber limitado en el pasado reciente la capacidad e incluso la necesidad de las instituciones financieras reguladas (incumbentes) de innovar y adaptarse a los cambios operados en la demanda. Los nuevos participantes están desempeñando un papel destacado en el fenómeno Fintech. Es cierto que las instituciones financieras tradicionales están adoptando paulatinamente los últimos avances tecnológicos, en gran parte como respuesta a la tensión competitiva de potenciales entrantes, pero también se está materializando esta entrada con nuevos agentes en la vanguardia del fenómeno Fintech, gracias a una mayor flexibilidad derivada de su carácter de «recién llegados» y a la necesidad de especializarse en determinados nichos de actividad o tecnologías. Ciertamente, aunque algunos de estos nuevos participantes son grandes compañías globales (por ejemplo, Amazon o Alibaba), muchas de ellas son startups relativamente pequeñas que operan en mercados regionales. La entrada de nuevos agentes podría cambiar considerablemente las estructuras de mercado actuales como resultado del desplazamiento, transformación e incluso desaparición de los intermediarios financieros incumbentes, e incluso no se puede descartar una reestructuración radical de la industria financiera en su conjunto (BIS, 2018).

\subsection{Oportunidades y retos de Fintech}

Dado su estado embrionario, aún no puede conocerse el efecto final que Fintech tendrá sobre los sistemas financieros. Por el momento solo pueden adivinarse ciertas oportunidades y retos que plantea el fenómeno Fintech, que se verán a continuación.

Entre las oportunidades, en primer lugar, Fintech constituye una doble innovación, tanto de proceso como de producto. Fintech es una innovación de proceso, pues en esencia permitiría una mejor explotación del input más importante de la industria financiera: la información. Esto conllevaría que se podría tanto explotar mejor la información que ya se venía analizando como empezar a explotar nueva información disponible que hasta la fecha carecía de utilidad comercial. Es más, la posibilidad de explotar mejor la información puede contribuir a reducir las asimetrías informativas que plagan la actividad financiera. Asimismo, Fintech puede representar una innovación de producto. En este sentido, puede ampliar la frontera de $\triangleright$ 
producción mediante la generación de nuevos productos o servicios que antes no estaban disponibles debido a los problemas de información señalados.

Como derivada de esta doble innovación se encontraría la «centralidad del cliente» (Nicoletti, 2017), esto es, la personalización o adaptación de los servicios financieros a preferencias y necesidades individuales de sus usuarios, gracias a que estas características pueden ser inferidas del comportamiento (como el historial de compras) y las características objetivas (como variables sociodemográficas) de los usuarios. En consecuencia, Fintech puede servir para ofrecer productos financieros con un conjunto de características más acorde o cercano a la variedad ideal del usuario.

En este sentido se puede subrayar el hecho de que algunos de los nuevos canales de financiación que suponen Fintech permitirían el nacimiento de muchas empresas emergentes innovadoras o startups, por ejemplo, en los sectores de TIC (datos) de vanguardia. Es decir, estas nuevas posibilidades de financiación pueden suponer una mayor competencia en diferentes industrias con efectos secundarios significativos en términos de mayor crecimiento económico y bienestar para el conjunto de la economía. Las TechFin (especialmente BigTech) juegan aquí un papel importante, ya que pueden partir con ventaja como innovadores de productos en el sector financiero. De hecho, estas empresas típicamente tienen acceso a información relevante sobre los clientes más allá del ámbito financiero y han acumulado la experiencia en el análisis y explotación de esta información, lo que puede mejorar la evaluación financiera de los clientes.

Asimismo, con Fintech, nuevos competidores están disputando los mercados a las instituciones financieras tradicionales. Esta mayor tensión competitiva puede fomentar la eficiencia gracias a una mayor disciplina de mercado. De hecho, la impugnabilidad en algunas actividades podría llevar a un cambio sustancial en la estructura de algunos sectores e incluso entidades, como la posibilidad de desagregación (unbundling) de las instituciones financieras, especialmente los bancos, desde las gigantescas corporaciones actuales (a menudo «demasiado grandes para caer» o too-big-to-fail) que prestan un amplio abanico de servicios hacia otras de menor tamaño. En este sentido, Fintech podría ayudar a aliviar el problema de riesgo moral de las instituciones de carácter sistémico.

Finalmente, Fintech puede fomentar la inclusión financiera, tanto en los países desarrollados como en los países en desarrollo. En los países industrializados, los clientes pequeños (hogares de ingresos bajos a medios y pymes) no suelen tener acceso a toda la gama de servicios financieros que los grandes clientes tienen a su disposición (Zetzsche, Buckley, Arner y Barberis, 2017), como, por ejemplo, la gestión de finanzas personales, por lo que las potenciales ganancias de eficiencia de Fintech se pueden materializar en una extensión de la demanda de servicios financieros cubierta. Además, este también puede ser el caso de los países en desarrollo, que generalmente carecen de mercados financieros confiables, lo que tiende a lastrar su desarrollo económico.

No obstante, Fintech también supone ciertos retos derivados en gran parte de la novedad que conlleva este tipo de negocios. En primer lugar, se plantean diversos riesgos desde el punto de vista de su efecto sobre la competencia. Estos riesgos en el terreno de la competencia están rodeados de cierta incertidumbre, pues se trata de situaciones relativamente novedosas sobre las que la investigación académica aún está desarrollándose. $\triangleright$ 
En concreto, algunos de los nuevos servicios Fintech se basan en plataformas digitales (como el crowdfunding) y redes, que presentan en la actualidad cierta tendencia a la elevada concentración e incluso a la supervivencia de una única plataforma como monopolista, merced a efectos complejos de los denominados mercados de múltiples lados (multisided markets), como los efectos de red directos y especialmente indirectos. Una derivada de esta digitalización creciente de los negocios (y no solo Fintech) es la posibilidad de aplicar, gracias a la ingente cantidad de información recopilada y al big data, precios personalizados, nuevo modo para referirse a la discriminación de precios, lo que genera ganadores (por el efecto «expansión del mercado») y perdedores (por el efecto «apropiación») entre los consumidores, cuyos efectos sobre el bienestar agregado, por tanto, difícilmente pueden determinarse (Bourreau y de Streel, 2018). Asimismo, el uso de algoritmos matemáticos por empresas Fintech en su operativa habitual puede representar una fuente de opacidad o falta de transparencia respecto al mecanismo lógico subyacente que explica la adopción de decisiones empresariales (incluidas las de precios), especialmente relevante cuando estas decisiones pueden resultar contrarias a la libre competencia, por ejemplo, si los algoritmos de varios competidores «aprenden» a coludir tácitamente (Ezrachi y Stucke, 2016). También se plantean riesgos en términos competitivos por la irrupción en los mercados financieros de grandes empresas como BigTech que puedan extender (leverage) un posible poder del mercado actual en sus mercados de origen a los mercados financieros en los que decidan introducirse.

En segundo lugar, existe un riesgo sobre la estabilidad del segmento de la actividad financiera cuyas empresas, por el descalce de plazos y riesgos con el que operan, son especialmente proclives a la inestabilidad: el sector bancario. Una mayor competencia en este segmento tendría efectos inciertos sobre la estabilidad de estas entidades tal como las conocemos hoy día, pues existe un intenso debate académico no resuelto sobre la interacción competencia-estabilidad (Vives, 2016). Podría darse que estos problemas de estabilidad se vieran incrementados en un primer momento en el conjunto de las actividades Fintech debido al mayor y más fácil acceso a clientes (internet móvil) que las nuevas tecnologías brindan a los nuevos negocios financieros. Este riesgo potencial vendría derivado tanto del hecho de tratarse de actividades financieras novedosas cuyos efectos agregados son, a día de hoy, desconocidos como de un posible aumento de la ocurrencia de comportamientos oportunistas e incluso directamente fraudulentos de los responsables de estos nuevos negocios, que podrían disfrazar de «mala suerte» una falta de diligencia o incluso un fraude. En este caso, y en ausencia de una regulación que lo aborde, los consumidores deberán en todo momento estar perfectamente informados y ser plenamente conscientes de los riesgos a los que se exponen cuando se adentran en el universo Fintech.

$Y$ finalmente, reiterando el proceso de digitalización creciente del sector financiero que posibilita el fenómeno Fintech, puede identificarse un mayor riesgo en términos de ciberseguridad, de modo que el sector financiero se haga más propenso a ciberataques y al cibercrimen. Esta preocupación da lugar a una nueva forma de regulación financiera dedicada a la salvaguarda de la estabilidad financiera en este ámbito (González-Páramo, 2017). 


\section{Derivadas sectoriales del fenómeno Fintech}

En este apartado se detallan seis ejemplos de innovaciones «disruptivas» $y$ «sustanciales» (y no meramente «incrementales») que están surgiendo dentro del ecosistema Fintech. De hecho, la idea de que sea más ilustrativa una aproximación por segmentos pone de manifiesto la ya aludida «disgregación» (unbundling) del sistema financiero. Las instituciones financieras tradicionales, que se basaban en un modelo de ventanilla única (one-stop shop) para proveer de manera integrada la mayoría de servicios financieros con el argumento de las economías de alcance, se ven amenazadas por competidores de nicho que se especializan en solo alguna(s) actividad(es), normalmente la(s) más rentable(s), realizando un «descreme» (cream-skimming) del mercado (IOSCO, 2017).

\subsection{Neobancos}

Los «neobancos» (neobanks o challenger banks) se pueden definir como entidades financieras con una interfaz netamente digital (aplicación móvil y página web) que prestan servicios propios del canal bancario (cuentas corrientes, depósitos y productos de ahorro, tarjetas de crédito y servicios de pago, descubiertos, asesoramiento financiero y préstamos, etcétera), aunque de una manera muy diferente a la tradicional (BIS, 2018):

- Los neobancos son entidades nacidas en el ecosistema digital, por lo que explotan su interoperabilidad con otras aplicaciones móviles y con redes sociales, mejorando la conveniencia y la experiencia para el usuario.
- Los neobancos destacan por el uso de tecnologías relacionadas con el big data, como los servicios en la nube (cloud computing) o el manejo de algoritmos, lo cual conlleva dos implicaciones por el lado de la oferta. En primer lugar, una estructura de costes más liviana en comparación con las infraestructuras heredadas y oficinas físicas de los operadores tradicionales, lo que implica que no necesitan un gran tamaño para ser rentables, pudiendo centrarse en nichos concretos (como los consumidores o las empresas pequeñas y medianas) o segmentos de mayor rentabilidad (como los pagos, los descubiertos o las operaciones en divisas) en comparación con el modelo de «ventanilla única» de los grandes bancos. En segundo lugar, una tecnología menos idiosincrática que puede utilizarse en otros ámbitos distintos al financiero, lo que puede dar entrada en la actividad bancaria a empresas de otros sectores tecnológicos (en el conocido como fenómeno TechFin) como las grandes plataformas digitales (BigTech). La explotación de datos y algoritmos también tiene una implicación por el lado de la demanda, como es la mayor capacidad de personalización de las condiciones del servicio, incluyendo el precio.

- Los neobancos suelen basar sus ingresos en comisiones (o incluso en la monetización de bases de datos) más que en la fuente tradicional del margen de intereses (que utilizan más los bancos tradicionales por su facilidad para captar financiación asequible por la extensión de depósitos).

Se trata, por tanto, de innovaciones interesantes en términos de competencia y eficiencia en el sistema bancario (FCA, 2018). Al $\triangleright$ 
centrarse en los nichos más rentables, disciplinan a los bancos tradicionales al dificultar estrategias de subsidios cruzados (consistentes en proveer ciertos servicios de manera gratuita, como las cuentas bancarias, sufragados con el elevado coste de ciertos servicios, como los descubiertos). No obstante, se ha de valorar su mayor potencial de inestabilidad al basarse en fuentes de ingresos menos diversificadas y menos estables (en comparación con la preponderancia de la captación de depósitos en el sector bancario tradicional).

\subsection{Tecnologías de registro distribuido}

Las tecnologías de registro distribuido (Distributed Ledger Technologies, DLTs) son una forma de mantener y actualizar un registro digital de transacciones de manera transparente mediante la validación de los participantes de la red, denominados nodos. Su utilidad para los mercados financieros (y en otros ámbitos) es evidente (McQuinn, Guo y Castro, 2016), pues permiten actualizar la propiedad a través de los datos relevantes de las transacciones: vendedor, comprador, precio, importe, fecha y hora, etcétera.

Existen dos tipos de DLTs: centralizadas o privadas y descentralizadas o públicas (Morales, 2018). Las primeras solo permiten, normalmente mediante invitación, la validación de transacciones y la actualización del registro a ciertos nodos participantes que actúan como «notarios del sistema». Las segundas, entre las que destaca la denominada tecnología de la cadena de bloques (blockchain), suponen un registro digital de transacciones mantenido y controlado de forma compartida y colectiva, donde la validación requiere algún tipo de mecanismo de consenso. Las primeras pueden ser, por tanto, más operativas, aunque las segundas pueden ser más disruptivas al eliminar en mayor medida los costes de transacción (pues no hay necesidad de compensar a los nodos centrales o notarios), algo fundamental en el sector financiero.

La aplicación de estas tecnologías va más allá de ser un registro, útil también para el cumplimiento normativo por la trazabilidad (Romero, 2018), pudiendo erigirse en auténticas facilitadoras de las transacciones. Así, destacan dos innovaciones que pueden ser catalizadoras de pagos y financiación.

Por el lado de los pagos, la utilización más popular e inicial de estas tecnologías ha sido la creación de monedas digitales/virtuales o criptomonedas. Estas representaciones digitales de valor (emitidas en general de forma privada y descentralizada) generan aspectos positivos por la reducción de costes de transacción en la validación y verificación de transacciones en conjunción con efectos de red (Catalini y Gans, 2016). Esto supone mayor competencia en los sistemas de pago, ampliando el volumen de transacciones reales y financieras (FMI, 2017), con un gran potencial en la financiación del comercio internacional (BIS, 2018) o incluso en los intercambios entre particulares.

Por el lado de la financiación destaca también el empleo de estas tecnologías mediante ofertas iniciales de monedas (ICOs, Initial Coin Offerings), que permiten a empresas (normalmente startups) captar fondos mediante la creación y emisión de sus propias monedas digitales (Catalini y Gans, 2018), en forma de fichas (tokens). Estas fichas (Gurrea y Remolina, 2018) pueden servir como medio de pago (payment tokens) para adquirir bienes o servicios de la empresa o la plataforma (utility tokens), o como un derecho a recibir parte de las ganancias o pagos de intereses futuros (security tokens).

Por tanto, criptomonedas e ICOs suponen una fuente de competencia en los sistemas $\triangle$ 
de pagos y en la financiación, lo cual puede implicar condiciones más ventajosas para las empresas necesitadas de estos servicios. Aunque estas tecnologías también pueden suponer un reto para el cumplimiento normativo o la organización de las transacciones, aparte del riesgo inherente de ciertos mecanismos de financiación como las ICOs.

\subsection{Revolución en los servicios de pago}

La disrupción en los servicios de pago puede ser quizás el elemento más paradigmático del fenómeno Fintech. Los nuevos proveedores que están entrando en este segmento van desde las grandes plataformas digitales (BigTech) hasta empresas de comercio minorista, de telecomunicaciones y manufactureras (Deutsche Bundesbank, 2016). La revolución incluye los ya conocidos monederos digitales, online o móviles. Pero también nuevos servicios como la iniciación de pagos (que permiten a un proveedor de bienes y servicios, con la autorización del cliente, obtener información de si se ha producido una orden de pago, de forma que la transferencia bancaria podría ganar cuota en el comercio electrónico respecto al pago por tarjeta) o la agregación de información sobre cuentas (que permiten acceder a información bancaria relevante a efectos de pagos o de otros servicios), auspiciados no solo por cambios tecnológicos, sino también regulatorios ${ }^{4}$ (Conesa, Gorjón y Rubio, 2018).

Estas innovaciones están aportando más competencia en el ámbito de los pagos, lo que

\footnotetext{
4 Fundamentalmente por iniciativas de «banca abierta» (open banking), que preconizan un acceso a datos en términos razonables y no discriminatorios, destacando la Directiva de Servicios de Pago, PSD2, Directiva (UE) 2015/2366 del Parlamento Europeo y del Consejo de 25 de noviembre de 2015 sobre servicios de pago en el mercado interior y por la que se modifican las Directivas 2002/65/CE, 2009/110/CE y 2013/36/UE y

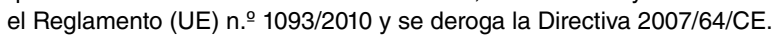

supone más y mejores servicios a un coste más asequible (WEF, 2017). Todo ello resulta bienvenido en un sector crítico para la competencia en todos los mercados, particularmente para pequeñas y medianas empresas y para aquellas involucradas en transacciones internacionales de comercio e inversión. También hay otras ganancias de eficiencia como la expansión de servicios bancarios y no bancarios por la explotación de los datos asociados a las transacciones. Aunque hay retos por las dificultades de nuevos entrantes en el acceso a datos que puedan ser útiles para prestar nuevos servicios o por el poder de mercado que pueden alcanzar grandes plataformas tecnológicas que se implanten en este sector.

\subsection{Nuevas formas de gestión y asesoramiento financiero}

La industria de asesoramiento y gestión de carteras también está sufriendo numerosos y profundos cambios cualitativos, destacando las siguientes innovaciones:

- La innovación más sencilla (y que es común a otros muchos sectores) es el desarrollo de comparadores financieros, que permiten confrontar la información de precios y otras condiciones de oferta mediante páginas web u otras herramientas digitales. Es una herramienta muy útil en servicios relativamente estandarizados (donde el precio, o tipo de interés, y otras condiciones pueden compararse fácilmente) y con demanda inelástica, lo que explica su aplicación a servicios financieros convencionales como los depósitos o el crédito hipotecario (y también a los seguros, como $\triangleright$ 
veremos más adelante), aunque también se extiendan a productos más sofisticados, como los fondos de inversión (Canada Competition Bureau, 2017).

- Una innovación adicional son los agregadores financieros, que aglutinan información de diferentes fuentes para explotarla y ofrecer servicios de interés para el consumidor final, ya sean servicios contables o financieros (desde el control de gastos hasta el asesoramiento y gestión de carteras), pero también otros servicios personalizados, como publicidad de determinados bienes y servicios (McQuinn, Guo y Castro, 2016).

- Las plataformas de networking y percepción (sentiment) llevan a cabo un análisis de investigación profesional sobre los mercados financieros mediante el uso de herramientas innovadoras como las redes sociales, donde se pueden intercambiar, compartir o incluso obtener ideas sobre la situación de los mercados financieros (IOSCO, 2017). A partir de ese análisis, y también utilizando las propias redes sociales, pueden ofrecer servicios de asesoramiento, intermediación y gestión.

- Una aproximación aún más innovadora es el denominado social trading (o copy trading), mediante el cual los agentes pueden seguir o imitar las decisiones de otros inversores que consideran mejor informados o más cualificados (AEFI, 2017). Por ello, estas plataformas también utilizan herramientas propias de redes sociales y, especialmente, los sistemas de evaluación y reputación, para señalizar a los mejores inversores (a imitar o seguir) y así generar confianza para el consumidor final.
- La última, y más disruptiva, innovación es la gestión y el asesoramiento automatizado o robotizado (robo-advisors) basado en inteligencia artificial (Deutsche Bundesbank, 2016). La voluntad de estas herramientas es atraer a un gran volumen de inversores para optimizar el funcionamiento de los algoritmos y además mejorar el binomio rentabilidadriesgo, mediante una puesta en común (pooling) y diversificación (spreading) del riesgo y un mayor uso de gestión pasiva, fondos ETF (exchange-traded funds) y fondos indexados (McQuinn, Guo y Castro, 2016).

Estas innovaciones tienen en común que mejoran la eficiencia estática, proveyendo servicios a un menor coste y generando fenómenos de inclusión financiera (al hacer más asequible el acceso a estos productos de asesoramiento y gestión). También mejoran la eficiencia dinámica al reducir fallos de mercado mediante la provisión de información (para limar asimetrías) o la separación de asesoramiento y gestión (para prevenir conflictos de intereses). Aunque hay que advertir que los productos pueden seguir siendo opacos y complejos para el consumidor final (McQuinn, Guo y Castro, 2016).

\subsection{Crowdfunding}

La financiación participativa (crowdfunding) se puede definir como la transferencia de fondos entre agentes (P2P, peer-to-peer) desde un amplio grupo de financiadores a un proyecto o empresa en particular (normalmente de un tamaño relativamente pequeño), generalmente a través de una plataforma digital (Wilson y $\triangleright$ 
Testoni, 2014). Aunque la financiación se puede aportar de manera no puramente lucrativa (vía donaciones o a cambio de ciertas «recompensas» para el consumidor como ediciones limitadas o premium de un bien, descuentos, etcétera), el segmento más relevante es la financiación con ánimo de lucro (crowdinvesting), tanto con la aportación de capital (equity) como especialmente mediante la aportación de préstamos. Actualmente, la financiación se está extendiendo incluso a otros productos, como la inversión inmobiliaria, la emisión de deuda en instrumentos de renta fija y el descuento de facturas.

La financiación participativa aporta una serie de ventajas: menores costes de transacción, externalidades de red al poner en contacto a múltiples inversores y proyectos en plataformas, explotación de la información para mejorar la calificación (scoring y rating) de proyectos (FMI, 2017; WEF, 2017) y posible reducción de riesgos por la puesta en común (pooling) y diversificación de proyectos (spreading). Por todo ello, este tipo de financiación puede beneficiar particularmente a empresas y proyectos nacientes, pequeños y medianos e innovadores, precisamente los más racionados en el mercado de crédito y los que más pueden dinamizar la competencia. Aunque se ha de tener en cuenta también los incentivos perversos de originar el riesgo para distribuirlo a continuación (originate to distribute) o los posibles sesgos de comportamiento (como conductas de rebaño) del consumidor (Deutsche Bundesbank, 2016).

\subsection{Insurtech}

El sector de los seguros también está experimentando su particular revolución digital, con distintas innovaciones en ciernes (Wilson, 2017):

- De nuevo, la innovación más sencilla son los comparadores (sitios web, aplicaciones móviles u otras herramientas digitales) para contrastar las condiciones de oferta de distintos productos, en términos de precios y coberturas. Estas herramientas son atractivas en los seguros, por su relativa estandarización y por el hecho de que su demanda venga inducida en muchas ocasiones por la propia regulación.

- Una innovación más disruptiva son los seguros entre particulares (P2P, peer-topeer). Esta desintermediación puede incrementar el margen para una mayor personalización de las condiciones y una reducción de fraudes y riesgos por el mayor contacto entre ambas partes.

- Otro segmento es la agrupación de consumidores en función de su nivel de riesgo (social brokering) para aplicar condiciones personalizadas y más favorables. La medición de riesgos a nivel individual y del hogar y, por tanto, las posibilidades de discriminación han aumentado por la generación de datos en el «internet de las cosas» (Internet of Things) y por la mejora en las técnicas de análisis.

- Finalmente, está el desarrollo de microseguros y seguros a demanda o ligados al uso o a transacciones específicas, muy unidos a los modelos de economía colaborativa.

Estos modelos suponen una desintermediación y una mayor eficiencia estática en costes. Pero también una mayor eficiencia dinámica por la mejora en la medición del riesgo $\triangleright$ 
(al utilizar tecnologías propias del big data) y una posible reducción del riesgo por su puesta en común (pooling) y diversificación (spreading) al adoptar modelos de plataforma que ponen en común a muchos agentes. Aunque estas tecnologías también abren la puerta a una mayor discriminación, que pueden chocar con principios de equidad (McQuinn, Guo y Castro, 2016).

\section{Conclusiones}

A lo largo del artículo, en nuestra aproximación general y sectorial, hemos podido constatar dos efectos fundamentales del fenómeno Fintech:

- Fintech conlleva un aumento de la competencia en el sistema financiero, lo que puede implicar más y mejores servicios, más personalizados y en condiciones más asequibles. Esto es una ventaja para los consumidores finales, pero también para las empresas que demandan servicios financieros como inputs intermedios, con lo que los efectos se dejarán sentir en toda la economía.

- Fintech puede mitigar fallos de mercado (sobre todo imperfecciones informativas), brindando una oportunidad para rebajar el tono restrictivo de la regulación sectorial.

En este sentido, la actuación de los poderes públicos debe ir encaminada a dar la bienvenida al mismo, diseñando un entorno regulatorio que maximice los beneficios potenciales del fenómeno, y no limitar su desarrollo salvo por razones de interés general o fallos de mercado debidamente justificados. Para fomentar la innovación, pueden ser bienvenidas medidas como un banco de pruebas regulatorio (sandbox), para testar el desarrollo de nuevos modelos de negocio; un hub de innovación, para un aprendizaje mutuo entre esos nuevos modelos y los reguladores y supervisores; y un enfoque funcional de la regulación, que ligue la regulación a los fallos de mercado asociados a cada actividad, independientemente de la forma jurídica de cada entidad (CNMC, 2018).

\section{Bibliografía}

[1] AEFI (2017). Libro Blanco de la Regulación Fintech en España.

[2] Arner, D. W., Barberis, J., y Buckley, R. P. (2017). FinTech, RegTech and the Reconceptualization of Financial Regulation. Northwestern Journal of International Law and Business, 37(3).

[3] Benston, G. J., y Smith, C. W. (1976). A Transactions Cost Approach to the Theory of Financial Intermediation. Journal of Finance, 31(2), 215-231.

[4] BIS (2018). Sound practices on the implications of Fintech developments for banks and bank supervisors.

[5] Bourreau, M., y De Streel, A. (2018). The regulation of personalised pricing in the digital era. Nota para la mesa redonda «Personalised Pricing in the Digital Era», de la OCDE (2018). Recuperado de www.oecd.org/daf/competition/ personalised-pricing-in-the-digital-era.htm

[6] Canada Competition Bureau (2017). Technology-led innovation and emerging services in the Canadian financial services sector.

[7] Catalini, C., y Gans, J. S. (2016). Some Simple Economics of the Blockchain. NBER Working Paper Series, 22952.

[8] Catalini, C., y Gans, J. S. (2018). Initial Coin Offerings and the Value of Crypto Tokens. MIT Sloan Research Paper, 5347-18.

[9] CNMC (2018). Estudio sobre el impacto en la competencia de las nuevas tecnologías en el sectorfinanciero(FINTECH).E/CNMC/001/18. D 
[10] Conesa, C., Gorjón, S., y Rubio, G. (2018). A new regime for access to payment accounts: THE PSD2. Financial Stability Review, Bank of Spain (35), 79-98.

[11] Deutsche Bundesbank (2016). Technologyenabled financial innovations: a source of opportunities and risks. Financial Stability Review, 67-77.

[12] Diamond, D. W. (1984). Financial intermediation and delegated monitoring. Review of Economic Studies, 51(3), 393-414.

[13] Ezrachi, A., y Stucke, M. E. (2016). Virtual Competition: The Promise and Perils of the Algorithm-Driven Economy. Cambridge: Harvard University Press.

[14] FCA (2018). Strategic Review of Retail Banking Business Models.

[15] FMI (2017). Fintech and Financial Services: Initial Considerations. IMF Staff Discussion Note SDN/17/05.

[16] Freixas, X., y Rochet, J. C. (2008). Microeconomics of banking. Cambridge. MA: MIT Press.

[17] González-Páramo, J. M. (2017). Financial innovation in the digital age: challenges for regulation and supervision. Revista de Estabilidad Financiera (32), 9-37.

[18] Gurrea, A., y Remolina, N. (2018, abril). Problemática jurídica y financiera de las Initial Coin Offerings (ICOs). Expansión. Recuperado dehttps://hayderecho.expansion.com/2018/04/ 11/problematica-juridica-y-financiera-de-lasinitial-coin-offerings-icos/
[19] IOSCO (2017). IOSCO Research Report on Financial Technologies (Fintech).

[20] McQuinn, A., Guo, W., y Castro, D. (2016). Policy Principles for Fintech. Information Technology \& Innovation Foundation.

[21] Morales, J. (2018). ¿Qué es blockchain? En García, P. Criptoderecho. La regulación de blockchain. Madrid: Wolters Kluwer España.

[22] Nicoletti, B. (2017). The Future of Fintech: Integrating Finance and Technology in Financial Services. Palgrave Macmillan (Palgrave Studies in Financial Services Technology).

[23] Romero, J. L. (2018). Tecnología de registros distribuidos (DLT): una introducción. Boletín Económico del Banco de España, 4/2018.

[24] Vives, X. (2016). Competition and Stability in Banking. The Role of Regulation and Competition Policy. Princeton: Princeton University Press.

[25] WEF (2017). Beyond Fintech: A Pragmatic Assessment Of Disruptive Potential In Financial Services.

[26] Wilson, J. D. (2017). Creating Strategic Value through Financial Technology. Hoboken: Wiley.

[27] Wilson, K., y Testoni, M. (2014). Improving the role of equity crowdfunding in Europe's capital markets. Bruegel Policy Contribution Issue 2014/09.

[28] Zetzsche, D. A., Buckley, R. P., Arner, D. W., y Barberis, J. N. (2017). From FinTech to TechFin: The Regulatory Challenges of Data-Driven Finance. EBI Working Paper Series (6). 